\title{
¿Cambios de rumbo? El gobierno Bolsonaro y la realidad politica de Brasil
}

\section{Path Change? Bolsonaros's government and politics in Brazil}

\section{Michelle Fernandez (D)}

Instituto de Ciencia Política/Universidad de Brasilia (IPOL/UnB)

michelle.fernandez@unb.br

\section{Humberto Dantas (iD}

Centro de Liderança Pública (CLP)

drhdantas@gmail.com

\section{Graziella Testa iD}

Escola de Políticas Públicas e Governo/Fundação Getúlio Vargas (EPPG/FGV) graziella.testa@fgv.br

\begin{abstract}
Resumen
¿Brasil vive una crisis de liderazgo politico? En este artículo, describimos cómo se organiza el gobierno de Jair Bolsonaro; sus conflictos internos y externos; la relación entre el Ejecutivo y el Legislativo y cómo esos factores influyen e impactan la dinámica de formulación de políticas públicas en el país. Para eso, trataremos de presentar y analizar las relaciones de ese gobierno con el Congreso Nacional, es decir, con la Cámara de Diputados y el Senado Federal. Analizamos las instituciones formales $e$ informales a partir de una descripción de los primeros dieciocho meses de gobierno. Al final, presentaremos la dinámica de construcción de politicas públicas del gobierno Bolsonaro, haciendo hincapié en el panorama de las politicas sociales en ese periodo.
\end{abstract}

Palabras clave: Brasil, instituciones, politicas sociales, gobierno, legislativo.

\section{Abstract}

Is Brazil facing a leadership crisis? In this article, we describe how Jair Bolsonaro's government is organized, with its internal and external conflicts. We also look at the relation between Legislative and Executive and how those factors impact on the public polices design dynamics in Brazil. Therewith, we present and analysis of the relations between this government and the Congress, both the Chamber of Deputies and the Senate. We analyze the formal and informal institutions of the government constructions in the first eighteen months of Bolsonaro's administrations. Finally, we present the dynamics of public policy construction of Bolsonaro's administration emphasizing the social policies during this period.

Keywords: Brazil, institutions, social policies, government, congress.

Articulo: Recibido el 30 de mayo de 2020 y aprobado el 19 de junio de 2020

\section{Cómo citar este artículo:}

Fernández, M., Dantas, H. \& Testa G. (2020) ¿Cambios de rumbo? El gobierno Bolsonaro y la realidad política de Brasil. Reflexión política 22(45), pp. 44-53. doi: https://doi.org/10.29375/01240781.3918 


\section{Introducción}

La joven democracia brasileña ha pasado por una serie de pruebas en los últimos años. Tras el fin de la dictadura militar, en 1985, pocas veces habíamos tenido un periodo en el que coincidieran importantes tormentas económicas y políticas a la vez. La pérdida de apoyo político del gobierno de Dilma Rousseff, la creciente crisis económica y la insatisfacción social han generado un escenario de inestabilidad política que ha dado lugar a la llegada de Bolsonaro al poder.

En octubre de 2018, los brasileños acudieron a las urnas para elegir un nuevo presidente de la República y para componer la Cámara de Diputados y el Senado Federal, además de elegir gobernadores de estado y diputados estaduales. Tras un pleito electoral muy polarizado, ganó las elecciones Jair Messias Bolsonaro. Con casi 30 años de carrera política, Bolsonaro nunca había ocupado lugar expresivo en el escenario político. Siempre había sido un parlamentario de "segunda línea" y se destacaba por posiciones políticas polémicas, radicales y cargadas de prejuicio.

Las características de la actuación de Bolsonaro en la Cámara de Diputados antes de ocupar la silla presidencial no dejaban claro cuáles serían los rumbos de la política brasileña después del 1 de enero de 2019. A pesar de los muchos años de vida pública llegaba al poder un outsider político. Además de eso, Brasil seguía polarizado bajo crisis económica y política. Así, contestar a algunas preguntas es importante para entender la realidad de Brasil a partir del 2019: ¿Cómo se organiza el gobierno Bolsonaro?; ¿cómo se da la relación del presidente con el Parlamento brasileño?; y ¿cuáles son las dinámicas de generación de políticas públicas? En las líneas que siguen, trataremos de presentar cómo se organiza el gobierno Bolsonaro a partir del análisis de las fuerzas políticas que componen el referido gobierno. Además de eso, trataremos de presentar y analizar las relaciones de ese gobierno con el Congreso Nacional, es decir, con la Cámara de Diputados y el Senado Federal. Por último, presentaremos algunos resultados políticos de los primeros dieciocho meses de gobierno, haciendo hincapié en el panorama de las políticas sociales en ese periodo.

\section{Mirando hacia dentro: cómo se organiza el gobierno Bolsonaro}

En los primeros meses del gobierno Bolsonaro, Dantas (2019) afirmó que la gestión actual sería "de" enfrentamiento y se mantendría "en" enfrentamiento. Eso se debe al hecho de que el presidente es históricamente adepto del conflicto y organizó un equipo de trabajo con diferentes líneas ideológicas que, desde el interior de los ministerios, son responsables por la generación de disputas internas que llevan a un absoluto desorden, o algo muy cerca de eso.

El escenario actual no es muy diferente de lo que se había imaginado en el 2019. Bolsonaro ha sido elegido por el Partido Social Liberal (PSL), pero dejó el partido después de poco más de 11 meses de mandato, en noviembre de 2019. Con menos de dos años en el poder, él es el presidente que colecciona la más numerosa cantidad de pedidos de impeachment desde el regreso de Brasil a la democracia, 31 pedidos. En un país que pasa por una intensa polarización política desde 2013, ese número de pedidos de impeachment puede no representar tanto, pero es necesario recordar que, en términos partidarios, Bolsonaro se ha elegido con la menor base de apoyo legislativo de la historia reciente de la nación. Entre los 513 diputados de la Cámara Federal de Brasil, su partido, el PSL, ha tenido poco más de 50 diputados federales, y su coalición electoral, formada por el PSL y el Partido Renovador Laborista Brasileño (PRTB) del vicepresidente Hamilton Mourão, no ha agregado ni un escaño para el grupo parlamentario.

El bajo apoyo parlamentario al gobierno Bolsonaro diseña un panorama de fragilidad para el gobierno. Aun así, Bolsonaro no ha buscado sistemáticamente diálogo público con los partidos que podrian ayudar, para aumentar su base de apoyo en el parlamento. Por el contrario, en las redes sociales y en entrevistas para los medios de comunicación tradicionales, el presidente ha repetido diversas veces que los partidos son corruptos y que su gobierno no negociaría para obtener victorias junto a los parlamentarios. Eso ha hecho que la agenda del Ejecutivo no avanzara en el Congreso Nacional en diferentes ocasiones. 
Además de eso, Bolsonaro imprime posiciones muy controvertidas en temáticas polémicas acerca de las costumbres y la cultura. $\mathrm{El}$ presidente se ha caracterizado en su ejercicio de gobierno por su actuación política conflictiva con representantes de diversos colectivos sociales, dentro de los que se destacan defensores de derechos humanos, ambientalistas, minorías étnicas e integrantes de la comunidad LGBTQ+. El presidente insiste en la defensa del armamento personal y viene afirmando, en estos casi 30 años de vida pública, que el Golpe Militar de 1964 fue una revolución que ha salvado al país del comunismo. En el encuentro de la Cumbre de las Naciones Unidas, en 2019, su discurso señaló el hecho de que su victoria en 2018 sacó el sesgo ideológico y puso fin a la dañosa izquierda del país.

A partir de las percepciones iniciales señaladas, es posible afirmar que Bolsonaro formó su gobierno basándose en cinco grupos de actores políticos distintos (Dantas, 2019). Son ellos:

1. Los militares: grupo que Anderson (2020) llama la fuerza más importante de sustentación de Bolsonaro, la base más estable y poderosa. Ha sido formado en los seis primeros meses del presidente en el poder por 45 personas en los más altos niveles. Actualmente, en 2020, tienen mucho espacio y poder en el gobierno y ocupan diversos ministerios importantes. Bolsonaro nunca negó su admiración por ese grupo. No se puede afirmar que los nombramientos de los militares no sean democráticos y previsibles. El problema es la obsesión del presidente por una falsa versión de la historia del país afirmando que los años de dictadura militar (1964-1985) fueron libertadores, revolucionarios, positivos y no rompieron con la democracia. El trauma generado en una parte de la sociedad contribuye en ese punto para una sensación constante de la posibilidad real de un nuevo golpe militar en el país.

2. Los conservadores: grupo que Almeida (2019) llama "ola conservadora". Está compuesto, sobre todo, por los nuevos evangelistas, caracterizados como liberales en términos económicos, reguladores en términos morales, intolerantes en términos sociales y más punitivos. Además de esos, la inspiración de los conservadores está en un astrólogo autodidacta en filosofía, tratado como escritor, Olavo de Carvalho, y en el hijo del presidente, Carlos Bolsonaro. Ambos opinan e influyen en el gobierno a partir de creencias anticientíficas.
Anderson (2020) afirma que la versión de la realidad del gobierno Bolsonaro, basada en guerras culturales, deja al margen los hechos políticos reales. Además, la izquierda, el feminismo y la homosexualidad son los enemigos principales, y la respuesta más clara son los homenajes a la dictadura, la defensa de los fabricantes de armas y de los militares en general. El "moralismo histérico" sería una característica importante de ese gobierno.

3. Los políticos: negando la política y los políticos, con la creación de una imagen falsa de su propio pasado, Bolsonaro eligió algunos representantes de esta clase para ocupar los ministerios. La decisión ha sido problemática, pues ha puesto en un mismo grupo actores implicados en corrupción, como el diputado y ministro Onix Lorenzoni (DEM-RS) y el exjuez y exministro de Justicia, Sergio Moro, conocido como un apóstol de la honestidad. Además, organizó su gobierno a partir de grupos temáticos en el Congreso Nacional, imaginando que tendría apoyo político a pesar de negarse a negociar con los partidos, que en el Legislativo brasileño dan muestras de una coordinación que no puede ser ignorada (Miranda, 2009). Sin embargo, en los últimos meses, durante la crisis generada por la pandemia de la COVID-19, Bolsonaro viene acercándose al Centrão. Ese grupo político ha sido cercano a todos los gobernantes que pasan por la silla presidencial a cambio de beneficios propios (Couto, Abrúcio y Texeira, 2014; Melo y Consentino, 2014). Parece ser que con Bolsonaro no va a ser diferente.

4. Los economistas: en la campaña electoral, Bolsonaro escogió al economista neoliberal Paulo Guedes como su representante para las temáticas económicas. Llegó a decir que era Guedes quien iba a dar todas las cartas en ese sector. De hecho, al asumir el poder ha creado un superministerio de Finanzas y entregado su comando a este aliado. El plan de Guedes ha sido, desde los primeros meses de gobierno, disminuir la presencia del Estado en las políticas públicas todo lo posible. Eso ha generado una disminución de las inversiones públicas, sobre todo en políticas sociales. El ministro y su equipo han tratado también de aprobar reformas que debilitan a los trabajadores, dejando a esos con cada vez menos apoyo del Estado, como ha sido la Reforma de la Seguridad Social. El mayor problema es que Guedes se muestra mucho más fiel a los preceptos neoliberales que Bolsonaro. Además de eso, el ministro viene teniendo dificultad para aprobar parte de su agenda por el Legislativo. 
5. Los juristas: la figura del exministro Sergio Moro, "el paladín" de la lucha contra la corrupción, fue el nombramiento más emblemático de Bolsonaro, por ese motivo su salida ha cambiado el equilibrio de fuerzas del gobierno. El exjuez es visto por sectores de la sociedad como un ícono de la moralidad y la acción contra políticos y empresarios involucrados en escándalos de corrupción en el país. Así, invitar a Moro fue una buena jugada para empezar el gobierno. Sin embargo, la figura de Moro ha estado constantemente en conflicto con la imagen de corrupción vinculada a las relaciones de la familia del Presidente con las milicias de Río de Janeiro (Anderson, 2020). Moro ha actuado en el gobierno bajo una agenda propia. Pero, sus proyectos en el Legislativo han sufrido derrotas expresivas, demostrando que él no tiene articulación política y que el gobierno Bolsonaro no tiene buenas relaciones con el Parlamento. Los enfrentamientos entre el exministro y el presidente se intensificaron, particularmente, el 24 de abril de 2020, cuando Bolsonaro exoneró al jefe de la Policía Federal en contra de la voluntad de Moro. Ese hecho ha llevado a la renuncia del exjuez a la carpeta ministerial y ha empezado una crisis de acusaciones y desconfianzas hacia el gobierno. Se cayó así el primer pilar, uno de los principales.

Entre los grandes desafios internos del gobierno Bolsonaro sobresale el problema de liderazgo. El presidente no tiene características de líder o gestor y nunca lideró un proceso político en sus 28 años como parlamentario en el Parlamento, donde cumplió siete mandatos sin ninguna expresión estratégica. Además de eso, el presidente no ha logrado aglutinar fuerzas políticas dentro del mismo partido que le eligió, el PSL. Ese no era un partido político grande antes de la llegada de Bolsonaro a la presidencia; por el contrario, era minúsculo. Sin embargo, en las elecciones de 2018, el PSL ha logrado elegir el presidente y un número significativo de diputados, transformándose como partido político. Por eso, cuando empezó el gobierno, muchos de los miembros del PSL no se conocían y no sabían si comulgaban los mismos valores y planes. Todo eso se podría ajustar con liderazgo y comunión, pero Bolsonaro no ha logrado hacerlo porque es una persona prepotente, principiante, obsesionada y errática (Anderson, 2020). Más allá de eso, hay una gran percepción de que Bolsonaro se acompaña de personas conflictivas como él, lo que pone al gobierno en la lógica de los enfrentamientos entre los miembros de su equipo.
Por lo tanto, la característica más emblemática de este gobierno es la capacidad de promover cambios caóticos en cargos vitales, inflamando simpatizantes en las redes sociales y dejándose llevar por ellos. El resultado es solo uno: el caos. Lo que queda por contestar es si ese caos es intencionado, es decir, es una estrategia del gobierno, o es una consecuencia de la falta de liderazgo de Bolsonaro. Todavía es muy pronto para decirlo con seguridad.

\section{Las relaciones con los otros poderes: cómo se desarrolla la relación Ejecutivo - Legislativo en el gobierno Bolsonaro}

La cuestión de la relación entre el Ejecutivo y el Legislativo juega un papel central en la literatura institucional brasileña porque se confunde con la cuestión de la estabilidad democrática. Entre los factores que resultaron en el golpe de Estado y el largo y violento período de la dictadura militar brasileña estuvo la difícil relación entre el Ejecutivo y el Legislativo en el período iniciado por la Constitución de 1946. La alta fragmentación del Congreso asociada con un Ejecutivo con poca capacidad para su propia agenda habría contribuido a la parálisis de decisión que hizo que fuera más propicio el golpe (Abranches, 2018; 1988).

Vale la pena recordar que las explicaciones ex post factum de los factores que dieron lugar al golpe de Estado en Brasil no son fatalistas, ni determinan que los mismos elementos necesariamente darían lugar al mismo escenario, pero ciertamente explican por qué la importancia de esta agenda de investigación y la gran cantidad y calidad de investigación en Brasil. Fueron estas interpretaciones del golpe y otros estudios internacionales en ingeniería constitucional los que caracterizaron a la academia de ciencias políticas de los años ochenta y noventa, generando una gran cantidad de predicciones para el conjunto de incentivos institucionales establecidos por la Constitución de 1988.

Sistemas electorales multipartidistas y proporcionales de lista abierta asociada con el presidencialismo resultarían en una parálisis de decisión constante. Esto se debe a que, en un sistema multipartidista, es muy raro que el jefe del partido de gobierno tenga la mayoría de los miembros del parlamento. En los parlamentarios, los impases se resolverían mediante instrumentos institucionales específicos para momentos en que el director ejecutivo no pudo llegar a un acuerdo. 
Sin embargo, en el presidencialismo, tales impases no serían solucionables ya que el jefe de gobierno es elegido por voto popular y no puede ser eliminado con justificación política, formalmente. A ese escenario, se agregó el federalismo que otorgó grandes poderes a los estados y municipios de la federación.

Sin embargo, las predicciones de parálisis no fueron confirmadas y los primeros estudios empíricos sobre el desempeño del Congreso en el contexto institucional en 1988 trataron de entender cómo se estaba resolviendo la ecuación de gobernanza. Se entendió que dos conjuntos de factores son fundamentales para que el Congreso y el Ejecutivo logren cierta armonía: la relación entre el presidente y los líderes del partido y la relación entre estos y sus respectivos bancos parlamentarios. En el primero, el período actual resultaría en una mayor centralización de la Agenda en manos del Ejecutivo, lo que sería consecuencia de los instrumentos de la Medida Provisional y la Urgencia Constitucional, dos incentivos institucionales que permitirian al Ejecutivo priorizar su agenda sobre la del Legislativo.

En el segundo, los datos empíricos (Figueiredo y Limongi, 1995; 1999; 2000) mostraron que los líderes del partido obtuvieron de sus escaños una alta disciplina en relación con la orientación de sus partidos, medida por un instrumento peculiar del parlamento brasileño, el de la orientación electoral. A través de este instrumento, los líderes del partido expresan la posición oficial del partido en los votos parlamentarios y es posible ver cuán disciplinados son los parlamentarios en términos de su liderazgo.

La explicación de la alta disciplina pasó por otros elementos microinstitucionales descuidados en la primera ola de estudios (Santos, 2003). Dos factores llevarian a los parlamentarios a votar de acuerdo con sus líderes en el plenario de la Cámara: 1) es por criterio del partido que el presidente elige a los ministros de Estado, por lo tanto, un parlamentario tendrá más probabilidades de ser nombrado ministro si está de acuerdo con su partido; 2) el presupuesto público en Brasil era lo que convencionalmente se llamaba autorización, lo que significa que el Congreso aprobaría el máximo que el Ejecutivo podría tener en recursos.

La última palabra sobre el presupuesto, la ejecución de recursos, sería prerrogativa de los ministerios. Por lo tanto, si un parlamentario está interesado en traer recursos a su distrito de origen, será necesario que él tenga una buena relación con su partido y este incentivo permitiría al Ejecutivo construir alianzas estables y una relativa previsibilidad en cuanto al número de votos que tendría disponible en el Congreso. En otras palabras, el presidente "forma su gobierno" cuando asigna miembros del partido para ocupar carteras ministeriales. Estos partidos, a cambio, apoyan la agenda del presidente ejecutivo en el Congreso.

Una tercera ola de literatura sobre las relaciones entre el Ejecutivo y el Legislativo trajo nuevas herramientas que nos ayudan a comprender. Freitas (2016) concluye que una buena parte de la agenda que se tomó como Ejecutivo en la segunda ola de literatura es en realidad la agenda de la coalición. Es decir, si el Ejecutivo puede ver su agenda aprobada, el proceso de formulación de esa agenda no ocurre sin la participación del Congreso, por el contrario, la construcción de esta agenda es compartida. Testa (2017) explora las otras formas de organización de los frentes y bancas legislativas, parlamentarias y comités parlamentarios, y concluye que no es posible ignorar el grado de formalización de las instituciones que permiten la construcción de la coalición.

North (1991) definió las instituciones como incentivos informales y reglas formales. Aun así, Helmke y Levitsky (2006) construyeron una clasificación de las instituciones informales en relación con las instituciones formales. Sin embargo, aunque las instituciones pueden ser tanto formales como informales, vale la pena señalar que una característica de las instituciones informales que no se puede descuidar es que no hay previsibilidad en cuanto al criterio para cambiarlas. Es decir, si el proceso legislativo de una enmienda constitucional, por ejemplo, tiene plazos, quórum y liturgia específica, el lastre institucional informal en el que se creó el presidencialismo de la coalición no tiene la misma seguridad. Por lo que, si los primeros treinta años se caracterizaron por presidentes y parlamentarios que optaron por tratar los incentivos institucionales formales de cierta manera, no existe una regla formal de que los próximos parlamentarios y presidentes hagan lo mismo.

Fue exactamente este punto el que salió a la luz después de las elecciones de 2018 con la elección de Jair Messias Bolsonaro. El proceso de construcción de coaliciones se asoció con 
prácticas corruptas en el discurso electoral, la elección de ministros de carteras ministeriales debería responder al criterio "técnico" de acuerdo con la campaña electoral de Jair Bolsonaro, y muchos otros. El discurso surge en un contexto de desacreditación de partidos y política.

$\mathrm{Si}$ el resultado de la ecuación de gobernabilidad en Brasil pasa necesariamente por este conjunto de instituciones informales y los partidos que resuelven los dilemas de la acción colectiva dentro del Legislativo Federal y entre el Ejecutivo y el Legislativo, la propuesta de Jair Bolsonaro sería formar su oficina con el personal técnico, a través de los partidos políticos, incluso si tales atributos, técnicos y políticos, no son mutuamente excluyentes. En la práctica, se intentó evitar a los partidos políticos y construir la coalición con los cinco grupos mencionados, militares, conservadores, economistas, abogados y políticos. Sin embargo, estos últimos no constituyeron relaciones sólidas de coaliciones, sino amistades extrapoladas para el campo político.

El presidente de la República, desde que asumió el cargo, se ha relevado de la tarea de construir una coalición. Es decir, encontrar partidos dispuestos a apoyar todo o parte de su agenda para tener cierta previsibilidad sobre el desempeño parlamentario. El presidente lo hizo porque la tarea de construir una coalición nunca fue una función formal del presidente de la República en Brasil. Por el contrario, los presidentes que lo han hecho han perdido puntos de popularidad al hacerlo. Por ejemplo, Lula, dependiendo del apoyo del PMDB para gobernar, veía a muchos votantes del PT insatisfechos. Así también, Fernando Henrique Cardoso, veía a los votantes socialdemócratas del PSDB muy descontentos cuando buscaba el apoyo del Partido del Frente Liberal. Jair Bolsonaro no estaba dispuesto a ver a algunos de sus electores insatisfechos para construir una coalición.

Sin embargo, las bases de apoyo del presidente están demostrando ser débiles. Los "políticos" amistosos no pueden operar la articulación política en el Congreso porque el presidente no está dispuesto a renunciar a nada; los conservadores siempre parecen estar más insatisfechos con la reducción de la influencia de Olavo de Carvalho en el gobierno y con los límites impuestos por el Congreso a propuestas absurdas; el máximo representante de juristas, Sergio Moro, deja al gobierno acusando directamente a Jair Bolsonaro de interferir en la Política Federal. Hay economistas y militares. El ejército, sin embargo, tiene su propia agenda económica.

Los economistas dirigidos por Paulo Guedes, Ministro de Economía de la Escuela de Chicago, intentan imponer una agenda de reducción del Estado en todas sus dimensiones con un enfoque en la privatización amplia y sin restricciones de las empresas estatales. La agenda económica militar desde los días de la dictadura ha estado más cerca de una perspectiva de desarrollo nacional. Con la crisis económica desencadenada por la pandemia Sars-CoV-2, surgió un plan económico para el "desarrollo de infraestructura" y la creación de empleo a través de la inversión estatal. El programa "Pro-Brasil" fue presentado por primera vez en 2019 por los ministros de Infraestructura y la Casa Civil, Tarcísio Freitas y el general Braga Neto, y fue convocado por el plan de medios "Marshall Tupiniquim". También hay similitudes entre el programa vinculado al ala militar y el programa principal de la presidenta del PT que sufrió un juicio político en medio de una crisis económica, Dilma Rousseff.

Estudios ya señalaban la dificultad de tratar las relaciones entre el Ejecutivo y el Legislativo en Brasil como si fueran dos actores univocos con agendas diferentes (Limongi, 2010). Esto se debe a que el Congreso estaría compuesto por varios grupos con diferentes intereses. A eso se agrega la posibilidad de un Ejecutivo también fragmentado que traiga viejas preocupaciones sobre la parálisis democrática.

\section{La dinámica de producción de los resultados políticos: las politicas públicas en el gobierno Bolsonaro}

En los apartados anteriores han sido señaladas características internas al gobierno Bolsonaro y algunas relaciones del gobierno con otros actores políticos que apuntan a la instauración del caos en el escenario político brasileño. Cuando se mira hacia las políticas públicas, sobre todo las políticas sociales, se nota que el actual presidente de Brasil, desde un principio, ha preparado su gobierno para ejecutar una parálisis en los avances sociales por medio de estos instrumentos. Políticas desarrolladas a lo largo de las últimas dos décadas están bajo serios riesgos (Arretche et al., 2019). 
Las políticas públicas sociales en el gobierno Bolsonaro han sido conducidas por medio de una dinámica basada en la austeridad. Para tratar de solucionar la crisis económica instaurada en el país, el nuevo presidente, asesorado por el ministro de finanzas Paulo Guedes, ha elegido actuar a partir de la disminución de la participación del Estado en una serie de políticas, principalmente en los temas sociales.

Esa reducción del Estado se basa en dos mecanismos. Por un lado, está la disminución de las actividades propiamente dichas del apoyo del Estado en temas sensibles a la ciudadanía, sobre todo en un contexto de grandes desigualdades sociales como es el caso de Brasil. Esa reducción brusca de intervenciones gubernamentales está vinculada a la idea de Estado minimo defendida por el actual gobierno. Bolsonaro deja claro que la preocupación por la desigualdad no es parte de las directrices de su gestión. El segundo mecanismo hace referencia al repaso de una serie de responsabilidades centradas en el gobierno nacional a los gobiernos subnacionales, sobre todo en términos de políticas sociales (Abrucio et al., 2020). Dejar de encargarse de políticas antes coordinadas por el gobierno central, poniéndolas bajo la responsabilidad de los gobiernos subnacionales, hace que Brasilia logre "ahorrar" todavía más, a cuestas del buen resultado de las políticas públicas.

Las elecciones políticas de Bolsonaro actúan en el sentido de vaciar las responsabilidades del gobierno federal en temas sociales, sobre todo en cuestiones de políticas de prestación de servicios como son educación y salud. Traspasar responsabilidades que son del gobierno central a los estados y municipios es actuar directamente para generar diferencias locales en términos de la oferta de servicios sociales. La reducción de acciones federales en esas políticas produce, sin lugar a duda, un aumento en las desigualdades entre regiones, ya que los gobiernos subnacionales tienen capacidades estatales distintas entre ellos.

Enfrentarse a problemas que profundizan las desigualdades en Brasil debería ser una agenda prioritaria para la clase política. Entre estos temas, la salud y la educación deben, necesariamente, colocarse en el centro de la discusión porque forman lo que podríamos llamar el núcleo duro de los derechos sociales. Sin embargo, durante los últimos años, los indicadores sociales en Brasil han mostrado un empeoramiento significativo. El discurso de austeridad económica predicado por el gobierno como una forma de avanzar hacia un nuevo proyecto de desarrollo ha afectado significativamente las políticas de salud pública y educación y, en consecuencia, ha impactado directamente en la calidad de vida de los ciudadanos. Las prácticas políticas en nombre de esta idea comenzaron a guiar al sector público en 2016, en el gobierno Temer, de manera estructurada con la aprobación de la Enmienda Constitucional 95, que impone una reducción de la presencia del Estado impidiendo el aumento de las inversiones estatales durante los próximos veinte años (Fernandez y Pellanda, 2018).

Además de eso, la administración del presidente Bolsonaro ha estado marcada por la falta de coordinación, desorganización y falta de experiencia en la gestión del sector público. En las áreas de salud, educación, cultura, ciudadanía y derechos humanos prevalecen decisiones ideológicas en una guerra cultural declarada a todo y a todos los que representan cualquier forma de conocimiento científico. Bolsonaro gradualmente impone su visión del mundo en la administración federal (Fernandes et al., 2020). Tanto la estructura del Ministerio de Salud, como la del Ministerio de Educación, materializan los deseos del presidente de promover una cultura militar y detener “ideologías comunistas”, que por lo general están asociadas con temas respaldados por la ciencia. Aparte del hecho de que la mayoría de las carteras ministeriales están formadas por generales de la reserva.

La situación más crítica del gobierno en términos de políticas sociales sucede en la crisis sanitaria de la COVID-19. La baja actuación del gobierno federal, la falta de coordinación más explícita y la actuación desde una perspectiva ideológica, sin tener en cuenta los aportes de la ciencia, han afectado el enfrentamiento a la pandemia.

Aunque el desempeño de los estados y municipios en el combate a la pandemia sea importante, es esencial reconocer la necesidad de la coordinación del gobierno federal en un plan conjunto para el país. Hay un contexto de asimetrías previas en las capacidades estatales, en recursos humanos y materiales, de las entidades subnacionales para implementar respuestas efectivas a la crisis impuesta por la epidemia. Además, una estrategia única para hacer frente a la pandemia, con las adaptaciones necesarias en la implementación local, 
es esencial para brindar respuestas satisfactorias a la crisis. En este momento, lo ideal sería tener la coordinación del gobierno federal combinada con el sólido desempeño de los gobiernos subnacionales (Fernandez, 2020). Además de eso, está puesta la importancia de considerar los conocimientos producidos por la ciencia para basar el proceso de toma de decisiones en términos de elección de la mejor opción para conducir el enfrentamiento de la crisis. Desafortunadamente, eso no ha sido considerado en Brasil. Hay también una discusión basada en ideología que viene conduciendo a la elección del mejor tratamiento para las personas infectadas por el COVID-19, el uso de la cloroquina. La elección del mejor tratamiento para los enfermos dejó de ser un tema discutido por la ciencia y la medicina y pasó a ocupar espacio en las discusiones político-ideológicas en todo el país.

De momento, e incluso antes de que empezara la pandemia de la COVID-19, los intentos del gobierno por mejorar la economía no han tenido éxito. Todos los "sacrificios" sociales para lograr la disminución de las cuentas públicas no han presentado datos que lleven a la conclusión de que la situación económica brasileña presenta señales de mejora. Lo que hay, sin embargo, es un empeoramiento de las cuestiones sociales. El impacto de la reducción de la inversión estatal en políticas sociales, como salud y educación ${ }^{1}$, el aumento de las tasas de desempleo ${ }^{2}$ y el consiguiente empobrecimiento de la población, llevan el país a la profundización de la vulnerabilidad social. Agregado a eso, la ineptitud del gobierno para llevar la máquina pública, el uso de una perspectiva ideológica en el proceso de toma de decisión y la desconsideración de los conocimientos razonables para la solución de los problemas públicos profundizan la crisis social del país. En pocos años, el país pasará por un proceso de empeoramiento de los indicadores sociales que han llevado algunas décadas en construcción. Brasil vive hoy una pandemia y un pandemonio.

\section{Apuntes conclusivos}

A lo largo del texto se ha tratado de señalar que el gobierno de Jair Bolsonaro está efectivamente marcado por conflictos internos y externos. La falta de diálogo del presidente con grupos políticos distintos de los suyos, los constantes cambios en las carpetas ministeriales, declaraciones abiertas $\mathrm{y}$ agresivas en las redes sociales contra varios sectores de la sociedad, conflictos con el Legislativo y con gobernadores y alcaldes, entre otros hechos, marcaron el escenario político brasileño en los primeros 16 meses del actual gobierno.

El fracaso en la construcción de mayorías sólidas en el Congreso Nacional muestra que Bolsonaro legisla a un ritmo similar a los otros presidentes, pero viene siendo derrotado como ningún otro antecesor suyo. Eso se debe a la incapacidad y falta de interés del presidente en mantener el diálogo con diferentes fuerzas políticas.

Durante casi año y medio en el poder, el presidente demostró ser errático en una serie de políticas públicas. Los resultados políticos generados por el gobierno no tienen interés en combatir la desigualdad, característica que marca las relaciones sociales en Brasil. Con una política económica basada en el Estado mínimo, no opta por inversiones en temas sociales. De la misma forma, el presidente deja de actuar para contemplar las necesidades de minorías políticas. Por lo tanto, políticas públicas de educación, salud y derechos humanos ya no están en la agenda del ejecutivo federal.

Además de eso, ante la pandemia, se desespera, niega la enfermedad, busca enfriar el impacto del aislamiento social al extremoy tiene entre sus seguidores y admiradores a quienes rechazan la existencia del virus. Critica abiertamente a los gobernadores y alcaldes, mostrando una absoluta falta de preparación para hacer frente a la crisis económica y social promovida por de la COVID-19. Brasil se enfrenta a una crisis de dimensiones importantes y tiene que lidiar con los improperios de un presidente al que poco le importa la situación de fragilidad humana que enfrentan los más de 200 millones de habitantes que tiene el país. Es evidente la crisis de liderazgo político reflejada en la incapacidad de Bolsonaro de concertar y coordinar la respuesta pública, amparándose en un discurso negacionista. Los rumbos de la sociedad brasileña bajo el mando de Bolsonaro son una incógnita que solamente el futuro va a revelar.

${ }^{1}$ De acuerdo con datos del Ministerio de Economía, en el presupuesto para 2020, el gasto de educación se ha reducido en el $16 \%$ y de salud en el 4,3\%. ${ }^{2}$ De acuerdo con los datos del Instituto Brasileño de Geografía y Estadística (IBGE), la tasa de desempleo en mayo de 2020 alcanzó el 11,8\%. 


\section{Referencias}

Abranches, S. (1988). Presidencialismo de coalizão: o dilema institucional brasileiro. Dados, 31(1), 5-34.

Abranches, S. (2018). Presidencialismo de coalizão: Raízes e evolução do modelo político brasileiro. São Paulo: Companhia das Letras.

Abrucio, F., Grin, E., Couto, C., Segatto, C. y Franzese, C. $(2020,12$ de abril). Mais Brasil, menos Brasília: o sentido do federalismo bolsonarista e seu impacto no combate à COVID-19. Jornal Estado de São Paulo.

Almeida, R. de (2019). Bolsonaro Presidente: conservadorismo, evangelismo e a crise brasileira. Novos Estudos Cebrap, 38(1), 185-21.

Anderson, P. (2020). Brasil à parte. São Paulo: Boi Tempo.

Arretche, M., Marques, E. y Faria, C. A. (2019). As políticas da política: desigualdades in inclusão nos governos do PSDB e PT. São Paulo: Editora UNESP.

Couto, C., Abrúcio, F. y Teixeira, M. (2014). De protagonista a parceiro da governabilidade: o papel ambíguo e indispensável do PMDB no sistema político brasileiro. En Dantas, H., Toledo, J. R. y Teixeira, M. (orgs.), Análise Política e Jornalismo de Dados - ensaios a partir do Basômetro. Rio de Janeiro: FGV Editora.

Dantas, H. (2019). Nas ondas de um governo de conflito, em conflito. In Fundação Konrad Adenauer, Boletim Brasil em Foco. Rio de Janeiro: Fundação Konrad Adenauer.

Fernandes, A., Teixeira, M. y Palmeira, J. (2020). A longa conjuntura crítica brasileira desde 2013: crise e castigo. Cadernos Gestão Pública e Cidadania, 25(81), 1-19.

Fernandez, M. (2020, 22 de mayo). Como as políticas de saúde deveriam ser conduzidas na pandemia. Nexo Jornal

Fernandez, M. y Pellanda, A. (2018). Austeridade e políticas sociais no Governo Temer: um panorama sobre as políticas de saúde e educação. En Monteiro, L. y Santana, L., Temerosas transações. Santa Cruz do Sul: Essere nel Mondo.

Figueiredo, A. y Limongi, F. (1995). Partidos políticos na Câmara dos Deputados: 1989-1994. Dados, 38(3), 497-524.

Figueiredo, A. y Limongi, F. (1999). Executivo e Legislativo na nova ordem constitucional (2a ed.). Rio de Janeiro: Editora FGV.

Figueiredo, A. y Limongi, F. (2000). Presidential Power, Legislative Organisation, and Party Behaviour in the Brazilian Legislature. Comparative Politics, 32(2), 151-170. DOI: https://doi. org/10.2307/422395
Freitas, A. M. de. (2016). Unboxing the Active Role of the Legislative Power in Brazil. Brazilian Political Science Review, 10(2), 1-34. DOI: https://doi. org/10.1590/1981-38212016000200004

Helmke, G. y Levitsky, S. (2006). Informal Institutions and Democracy: Lessons from Latin America. Baltimore: Johns Hopkins University Press.

Limongi, F. (2010). Horizontes das ciências sociais no Brasil: ciência política. Estudos legislativos, 1, 163-190.

Melo, C. y Consentino, L. (2014). Dinâmica do presidencialismo de coalizão: contribuição e limites do "Basômetro". En Dantas, H., Toledo, J. R. y Teixeira, M. (orgs.). Análise Política e Jornalismo de Dados - ensaios a partir do Basômetro. Rio de Janeiro: FGV Editora.

Miranda, G. L. (2009). A influência da arena eleitoral sobre o comportamento dos partidos políticos na Câmara dos Deputados e no Senado Federal. Dados, 52(4), 911-959. DOI: https://doi.org/10.1590/ S0011-52582009000400004

North, D. (1991). Institutions. The Journal of Economic Perspectives, 5(1), 97-112. DOI: https://doi. org/10.1257/jep.5.1.97

Santos, F. (2003). O poder legislativo no presidencialismo de coalizão. Belo Horizonte: Editora UFMG.

Testa, G. G. (2017). Informal Institutions and Coalitional Presidentialism in Brazil. Tesis de Doctorado, Departamento de Ciencia Política, Universidad de São Paulo, São Paulo. 